\section{Kontralateraler Zweittumor nach Bestrahlung}

Die Radiotherapie eines Mammakarzinoms verstärkt auch für das Risiko für ein zweites primäres Mammakarzinom. Dabei spielen offenbar der Zeitpunkt der Bestrahlung, hormonelle und reproduktive Faktoren eine Rolle.

$\mathrm{H}$ eute gelten Eintrittsalter in die Menarche, Anzahl der Schwangerschaften und Alter bei der ersten Schwangerschaft als etablierte Faktoren, die das Brustkrebsrisiko beeinflussen. So erniedrigt sich für Frauen mit einer ersten Schwangerschaft in jungen Jahren das Lebenszeitrisiko für ein Hormonrezeptor-positives Mammakarzinom. Als etablierter Risikofaktor gilt auch die Exposition gegenüber ionisierender Strahlung. In Phasen erhöhter Zellteilungsaktivität im Brustparenchym, etwa während $\mathrm{Pu}$ bertät oder Schwangerschaft, scheint die Suszeptibilität besonders hoch zu sein.

Im Rahmen der WECARE (Women's Environmental Cancer and Radiation Epidemiology)-Studie wurde der Einfluss reproduktiver und hormoneller Faktoren vor, während oder nach Diagnose eines Primärtumors in der Brust auf das Risiko für ein strahleninduziertes zweites primäres kontralaterales Mammakarzinom (CBC) untersucht. In diese multizentrische, bevölkerungsbasierte Studie waren 708 Frauen mit einem asynchronen $\mathrm{CBC}$ und 1.399 Frauen mit einem unilateralen Mammakarzinom (UBC) als Kontrollgruppe eingeschlossen.

Bei Erstdiagnose waren die Frauen in beiden Gruppen median 46 Jahre alt. Für Frauen, die zum Zeitpunkt der Erstdiagnose eines Primärtumors nullipar und im Rahmen der Radiotherapie des Primärtumors parallel einer Dosis von $\geq 1$ Gy im Bereich der kontralateralen Brust ausgesetzt waren, erhöhte sich das Risiko der Entwicklung eines strahlen- induzierten $\mathrm{CBC}$ im Vergleich zu analog Behandelten, die vor der RT ein Kind geboren hatten. Dieser Vorteil nivellierte sich für RT-behandelte Frauen, die später -im Zeitraum zwischen Erstdiagnose und dem Referenzdatum - schwanger wurden. Dann erhöhte sich das CBC-Risiko signifkant gegenüber nicht bestrahlten Patientinnen. Die Autoren fanden keine Korrelation zwischen CBCRisiko und Anzahl der Schwangerschaften, Stillzeiten oder menopausalem Status zum Zeitpunkt der Erstdiagnose.

Fazit: Das Risiko, nach Bestrahlung eines primären Mammakarzinoms ein $\mathrm{CBC}$ zu entwickeln, ist für nullipare Frauen erhöht. Trotz kleiner Fallzahlen scheint auch eine spätere Schwangerschaft nach Erstdiagnose das CBC-Risiko zu erhöhen. Wolfgang Zimmermann

Brooks JD et al. Reproductive status at first diagnosis influences risk of radiation-induced secondary primary contralateral breast cancer in the WECARE study. Int J Radiat Oncol Biol Phys. 2012;84(4):917-24.

\title{
Endokrine Therapie bei Brustkrebs: Patientinnen mit Gelenkschmerzen leben länger
}

\section{Die klinische Wirksamkeit bestimmter Arzneimittel lässt sich am Auftreten spezifischer Nebenwirkungen ablesen. Dieser Zusammenhang gilt offenbar auch für die adjuvante endokrine Therapie des Mammakarzinoms.}

\footnotetext{
7 ur Minderung des Rezidivrisikos wird in internationalen Leitlinien bei postmenopausalen Frauen mit einem Hormonrezeptor-positiven Mammakarzinom im Frühstadium eine adjuvante endokrine Therapie empfohlen. Neben dem Tamoxifen rücken zunehmend Aromatasehemmer wie Exemes$\tan$ in den Vordergrund, die sich hinsichtlich der Wirksamkeit Tamoxifen als ebenbürtig und in einigen Studien sogar als überlegen erwiesen haben. Entsprechend der verschiedenen Wirkansätze unterscheiden sich auch die Nebenwirkungsprofile. So wird Tamoxifen mit gynäkologischen Nebenwirkungen, aber auch thromboembolischen Ereignissen in Verbindung gebracht. Unter Aromatasehemmern dominieren hingegen muskuloskeletale
}

Symptome. Für einige Substanzen wurden in der Vergangenheit bestimmte Nebenwirkungen als Zeichen für gute Wirksamkeit interpretiert. In der multinationalen Phase-III-Studie TEAM (Tamoxifen Exemestane Adjuvant Multinational) wurden über fünf Jahre Exemestan und die Sequenz TamoxifenExemestan beim ER/PR-positiven Mammakarzinom verglichen. Die abschließende Analyse nach fünf Jahren ergab keine signifikanten Wirksamkeitsunterschiede zwischen beiden Regimes. Als Hauptgrundlage für die Therapieentscheidung sollte allein das $\mathrm{Si}$ cherheitsprofil dienen.

In der vorliegenden Studie interessierte daher die Frage nach einem möglichen Zusammenhang zwischen bestimmten Nebenwirkungen und Thera- pieeffizienz. Dazu wurden retrospektiv die Daten der deutschen TEAM-Kohorte analysiert (739 mit der Sequenz Tamoxifen/Exemestan, 763 mit Exemestan-Monotherapie). Patientinnen mit therapiebedingter Arthralgie/Myalgie und menopausalen Symptomen wiesen ein signifikant längeres Gesamt- (OS) und krankheitsfreies Überleben (DFS) auf als Frauen ohne diese Nebenwirkungen - unabhängig vom Therapieregime. Signifikante Vorteile im DFS wurden nur bei Exemestan-behandelten Patientinnen mit den geschilderten Nebenwirkungen beobachtet.

Fazit: Nebenwirkungen wie Arthralgie/ Myalgie oder menopausale Symptome unter einer adjuvanten endokrinen Therapie des Hormonrezeptor-positiven Mammakarzinoms sind mit einem signifikant verbesserten Gesamtüberleben verbunden. Wolfgang Zimmermann

Hadji P et al. Correlation of treatment-emergent adverse events and clinical response to endocrine therapy in early breast cancer: a retrospective analysis of the German cohort of TEAM. Ann Oncol. 2012;23(10):2566-72. 